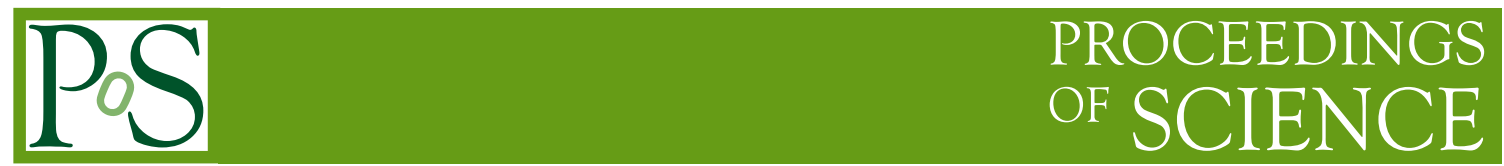

\title{
NSI at Hyper-Kamiokande
}

\section{Osamu Yasuda*}

Tokyo Metropolitan University

E-mail: yasuda@phys.se.tmu.ac.jp

In this talk we discuss the possibility to test a hypothesis, which explains the tension between the mass-squared differences of the solar neutrino and KamLAND experiments from the non-standard flavor-dependent interaction in neutrino propagation, by atmospheric neutrino observations at the future Hyper-Kamiokande experiment.

Neutrino Oscillation Workshop

4 - 11 September, 2016

Otranto (Lecce, Italy)

\footnotetext{
*Speaker.
} 


\section{Introduction}

Recently it was pointed out in Ref. [1] that there is a tension between the mass-squared difference deduced from the solar neutrino observations and the one from the KamLAND experiment, and that the tension can be resolved by introducing the flavor-dependent neutrino non-standard interaction (NSI) in neutrino propagation:

$$
\mathscr{L}_{\mathrm{eff}}^{\mathrm{NSI}}=-2 \sqrt{2} \varepsilon_{\alpha \beta}^{f f^{\prime} P} G_{F}\left(\bar{v}_{\alpha L} \gamma_{\mu} v_{\beta L}\right)\left(\bar{f}_{P} \gamma^{\mu} f_{P}^{\prime}\right)
$$

NSI in neutrino propagation modifies the matter potential in neutrino propagation. The atmospheric neutrino experiments cover a wide range of the neutrino energy and the baseline length of the neutrino path, so the oscillation probability involves the three neutrino mass eigenstates. The effective Hamiltonian in the case of atmospheric neutrino oscillations is given by

$$
H^{\mathrm{eff}}=\frac{1}{2 E} U \operatorname{diag}\left(0, \Delta m_{21}^{2}, \Delta m_{31}^{2}\right) U^{-1}+\sqrt{2} G_{F} N_{e}\left(\begin{array}{ccc}
1+\varepsilon_{e e} & \varepsilon_{e \mu} & \varepsilon_{e \tau} \\
\varepsilon_{\mu e} & \varepsilon_{\mu \mu} & \varepsilon_{\mu \tau} \\
\varepsilon_{\tau e} & \varepsilon_{\tau \mu} & \varepsilon_{\tau \tau}
\end{array}\right)
$$

where $\varepsilon_{\alpha \beta} \equiv \sum_{f=e, u, d ; P}\left(N_{f} / N_{e}\right) \varepsilon_{\alpha \beta}^{f f P} \simeq \sum_{P}\left(\varepsilon_{\alpha \beta}^{e e P}+3 \varepsilon_{\alpha \beta}^{u u P}+3 \varepsilon_{\alpha \beta}^{d d P}\right)$. On the other hand, in the case of the solar neutrino and KamLAND experiments, the oscillation probability involves only the two neutrino mass eigenstates to a good approximation, and the effective Hamiltonian in the case of atmospheric neutrino oscillations is given by

$H^{\mathrm{eff}}=\frac{\Delta m_{21}^{2}}{4 E}\left(\begin{array}{cc}-\cos 2 \theta_{12} & \sin 2 \theta_{12} \\ \sin 2 \theta_{12} & \cos 2 \theta_{12}\end{array}\right)+\sqrt{2} G_{F} N_{e}\left(\begin{array}{cc}c_{13}^{2} & 0 \\ 0 & 0\end{array}\right)+\sqrt{2} G_{F} N_{e} \sum_{f=e, u, d} \frac{N_{f}}{N_{e}}\left(\begin{array}{cc}-\varepsilon_{D}^{f} & \varepsilon_{N}^{f} \\ \varepsilon_{N}^{f *} & \varepsilon_{D}^{f}\end{array}\right)$.

The dimensionless coefficients $\varepsilon_{D}^{f}$ and $\left|\varepsilon_{N}^{f}\right|$ stand for the effects of NSI and can be expressed in terms of $\varepsilon_{\alpha \beta}$ and the standard oscillation parameters [1]. In this talk we discuss the possibility to test the hypothesis in Ref. [1] by atmospheric neutrino observations at HK. This talk is based on Ref. [2], and the details can be found in Ref. [2].

\section{Results and Conclusion}

We assume that the Nature is described by the standard three flavor neutrino framework, and our strategy is to see how significant the non-zero NSI effects can be in the atmospheric neutrino data at HK. For simplicity we consider the case of $f=d$, i.e., we assume that NSI affects only $d$ quarks. This is because the density profiles of $u$ and $d$ quarks are different in the Sun. For each point in the $\left(\varepsilon_{D}^{d},\left|\varepsilon_{N}^{d}\right|\right)$-plane, we compute $\chi^{2}$ as a function of all the $\varepsilon_{\alpha \beta}$ parameters as well as the standard oscillation parameters which affect the atmospheric neutrino oscillation probability, and marginalize $\chi^{2}$ with respect to these parameters. The allowed region is shown in Fig.1, where the superscript $f=d$ is dropped: $\varepsilon_{D, N} \equiv \varepsilon_{D, N}^{d}$. From Fig.1, if there are no non-standard interactions in nature, then we can conclude that the best fit point of the combined analysis of the solar neutrino and KamLAND data by Ref. [1] can be excluded at more than $11 \sigma(8 \sigma)$ in the case of the normal 
(inverted) hierarchy, while the best fit point of the global analysis in Ref. [1] can be excluded at $5.0 \sigma(1.4 \sigma)$ in the case of the normal (inverted) hierarchy.

It is remarkable that the tension, which was found in the low energy neutrino data $\left(E_{v} \sim\right.$ several $\mathrm{MeV}$ ) of the solar and KamLAND experiments, ${ }^{1}$ can be tested by the high energy neutrino data $\left(E_{V} \sim \mathscr{O}(10) \mathrm{GeV}\right)$ in the future atmospheric neutrino experiments with high statistics through the matter effect.
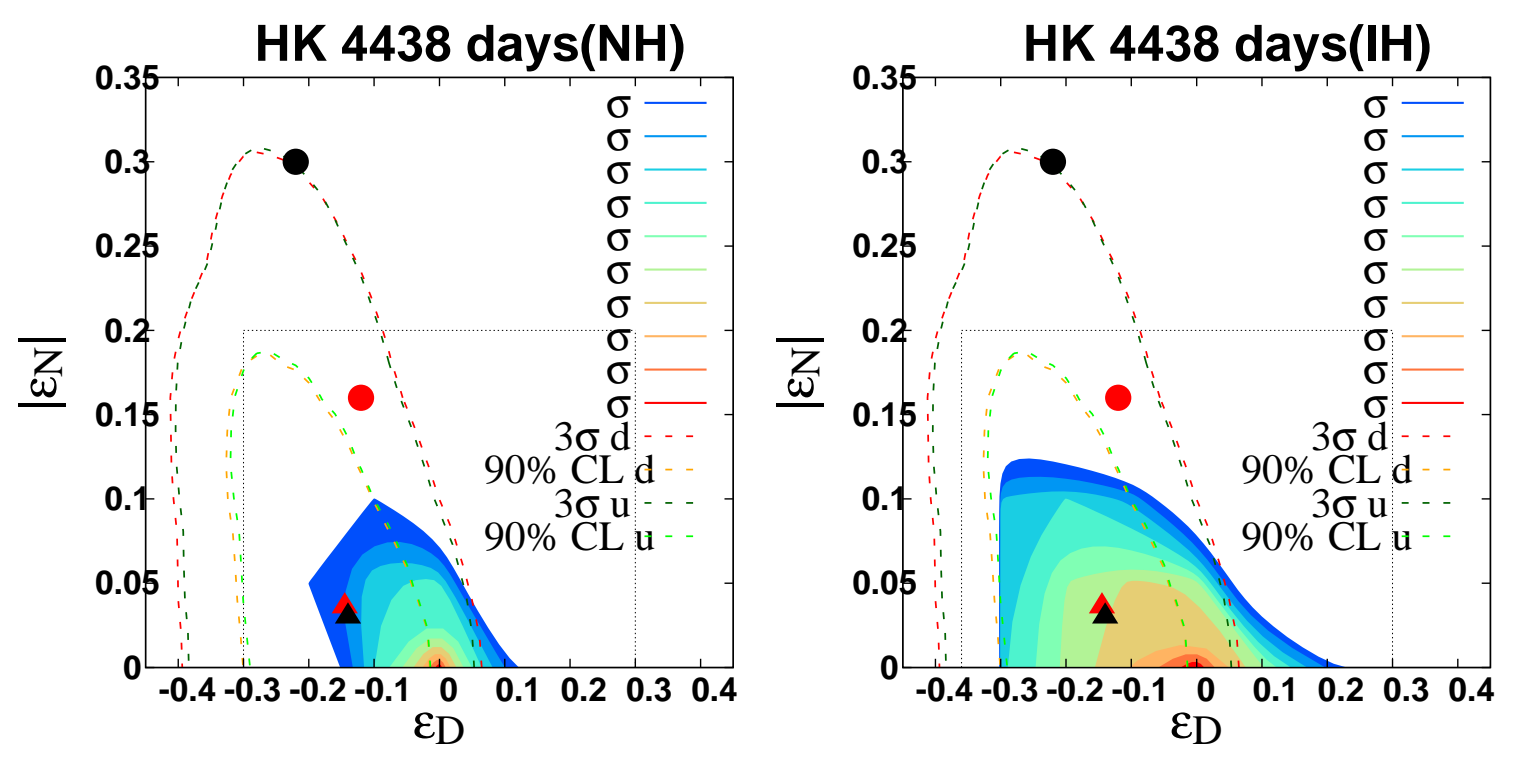

Figure 1: The allowed region in the $\left(\varepsilon_{D},\left|\varepsilon_{N}\right|\right)$ plane from the $\mathrm{HK}$ atmospheric neutrino data for the normal hierarchy (left panel) and for the inverted hierarchy (right panel). We calculated $\chi^{2}$ for $\left(\varepsilon_{D},\left|\varepsilon_{N}\right|\right)$ inside the area surrounded by dotted lines and at the best fit points. The red $(f=d)$ and black $(f=u)$ circles indicate the best fit point from the solar neutrino and KamLAND data [1] for NSI with $\left(\varepsilon_{D}^{d}, \varepsilon_{N}^{d}\right)=(-0.12,-0.16)$ (red) and that for NSI with $\left(\varepsilon_{D}^{u}, \varepsilon_{N}^{u}\right)=(-0.22,-0.30)$ (black), respectively. The red and black triangles indicate the best fit value from the global neutrino oscillation experiments analysis [1] for NSI with $\left(\varepsilon_{D}^{d}, \varepsilon_{N}^{d}\right)=$ $(-0.145,-0.036)$ (red) and that for NSI with $\left(\varepsilon_{D}^{u}, \varepsilon_{N}^{u}\right)=(-0.140,-0.030)$ (black), respectively. The dashed lines are the boundaries of the allowed regions from the global neutrino oscillation experiments analysis. For reference, we plotted for both the cases with $f=u$ and $f=d$.

\section{Acknowledgments}

This research was partly supported by a Grant-in-Aid for Scientific Research of the Ministry of Education, Science and Culture, under Grants No. 25105009, No. 15K05058, No. 25105001 and No. 15K21734.

\section{References}

[1] M. C. Gonzalez-Garcia and M. Maltoni, JHEP 1309 (2013) 152 [arXiv:1307.3092 [hep-ph]].

[2] S. Fukasawa and O. Yasuda, Nucl. Phys. B 914 (2017) 99 [arXiv:1608.05897 [hep-ph]].

[3] T. Kajita, in these proceedings.

\footnotetext{
${ }^{1}$ See Ref. [3] for the prospect of observation of the same effect by solar neutrinos at HK.
} 